\title{
Recent advances in spinal cord neurology
}

Dietz, V

\begin{abstract}
This short review summarizes developments and achievements made during the last few years in spinal neurology and includes all relevant papers published in the Journal of Neurology during this time. A focus of the review concerns the debate about the significance of translational medicine in spinal cord injury with the introduction of new drugs directed to achieve some spinal cord repairs.
\end{abstract}

DOI: https://doi.org/10.1007/s00415-010-5700-7

Posted at the Zurich Open Repository and Archive, University of Zurich

ZORA URL: https://doi.org/10.5167/uzh-35711

Journal Article

Published Version

Originally published at:

Dietz, V (2010). Recent advances in spinal cord neurology. Journal of Neurology, 257(10):1770-1773.

DOI: https://doi.org/10.1007/s00415-010-5700-7 


\title{
Recent advances in spinal cord neurology
}

\author{
Volker Dietz
}

Received: 15 June 2010/ Accepted: 2 August 2010/Published online: 18 August 2010

(C) Springer-Verlag 2010

\begin{abstract}
This short review summarizes developments and achievements made during the last few years in spinal neurology and includes all relevant papers published in the Journal of Neurology during this time. A focus of the review concerns the debate about the significance of translational medicine in spinal cord injury with the introduction of new drugs directed to achieve some spinal cord repairs.
\end{abstract}

Keywords Spinal cord repair - Myelopathy $\cdot$ Babinski sign $\cdot$ Neuroplasticity $\cdot$ Spinal cord ischemia

\section{Introduction}

Para-tetraplegia is the consequence of damage to the spinal cord from a trauma or non-traumatic (inflammatory, vascular, neoplastic or metabolic) origin with an acute or chronic-progressive appearance. The neurological deficits include motor, sensory and autonomic functions. The consequences are motor (spastic para-tetraplegia or flaccid paraparesis due to a cauda lesion), sensory (spinal sensory level with hyperaesthesia-algesia or anesthesia-algesia below the level of lesion) and autonomic (neurogenic bladder- bowel-, sexual-, and circulatory disturbances) dysfunction. Therefore, below the level of spinal cord injury (SCI), complex clinical disturbances exist. The severity of deficits depends on the level and completeness of the spinal cord lesion.

V. Dietz $(\bowtie)$

Spinal Cord Injury Center, Balgrist University Hospital, Forchstr 340, 8008 Zürich, Switzerland

e-mail:vdietz@paralab.balgrist.ch
This review summarises progress in spinal cord neurology and will focus on trauma and pathology concerning primarily the spinal cord, i.e., it will exclude the spinal cord involvement in systemic diseases as, for example, autoimmune or genetically determined diseases which also can involve the spinal cord.

After dealing with some aspects of spinal cord neurology, this review will focus on a discussion concerning spinal cord repair and the actual state of regenerationinducing therapies cf. [1]. Surprisingly, in specialized Journals such as 'Spinal Cord', the subject of spinal cord neurology plays only a minor role e.g., [2].

\section{Actual diagnostic approaches}

The long-term course of non-traumatic acute/subacute myelopathies is important to assess in follow-up examinations for recognizing etiology and prediction of outcome [3]. It is reported that an unfavorable outcome can be expected when the initial symptoms are severe, the lesion is centrally located in the spinal cord (MRI) and the etiology is neuromyelitis optica or part of a systemic disease. Over half of the patients subsequently develop multiple sclerosis, and in one-third the etiology at the end of the follow-up differs from that suspected initially. An intramedullary spinal cord hemorrhage is a quite uncommon cause of myelopathy and can present in an acute, subacute, or chronic fashion. Spinal vascular malformations (e.g., cavernomas) are the most common cause of such a hemorrhage [4].

The question concerning the presence or absence of the Babinski sign in severe spinal cord injuries is of clinical relevance. In only about half of the subjects suffering a complete SCI is the Babinski sign positive [5]. While the 
occurrence of the Babinski sign does not depend on the level of lesion, it is associated with high spastic muscle tone. In contrast, subjects with a negative Babinski sign show a low level, or absent, muscle tone. The low muscle tone can be attributed to peripheral nerve damage, myelomalacia below the level of lesion, or Baclofen therapy.

The clinical and imaging features in spinal cord ischemia are shown to be frequently connected with aortic disease and associated with a vertebral body infarction [6]. Concomitant spinal cord and vertebral body infarctions are frequently located in the thoracolumbar region. The neurological deficit in most of these subjects has a poor outcome, especially concerning their walking ability.

Rare cases of spinal cord damage due to sarcoidosis [7] and secondary to psoriatic arthritis [8] have been reported. Spinal cord sarcoidosis can successfully be detected using18F-FDG PET uptakes, an abnormality that resolves following clinical improvement with treatment. Acute transverse myelitis due to psoriatic arthritis is considered to be a variant of rheumatic arthritis with the rheumatoid factor being negative.

Two widely unknown clinical signs can be of diagnostic relevance in spinal cord pathology, Beevor's sign [9] and the painful neck rotation sign [10]. In Beevor's sign, an upward deflection of the umbilicus on flexion of the neck is the due to the paralysis of the inferior portion of the abdominis muscle. This condition can be caused by a SCI at or below the level of Th10 or, alternatively, by a facioscapulohumeral muscular dystrophy [9]. However, this sign is reported not to be as sensitive as previously assumed.

In patients suffering cervico-occipital pain, a painful and limited neck rotation may be indicative of the crowned dens syndrome, defined as acute neck pain due to deposition of crystals surrounding the odontoid process [10]. In these cases computed tomography shows mottled calcification around the odontoid process.

\section{Therapeutic approaches}

Novel therapies in spinal cord damage are rare. The administration of Erythropoietin (EPO) in SCI as a neuroprotective drug, blocking apoptosis, is discussed [11]. There are promising preclinical results. However, a clear demonstration of an effective pharmacological treatment is yet lacking. Thus, at present no therapy is available to directly limit spinal cord damage after trauma.

In incomplete SCI subjects, the exploitation of innate neuronal plasticity using a functional training programme is well established [12]. Neuroplasticity plays an important role in the neurorehabilitation even of elderly SCI subjects [13]. The progress made in this field concerns the definition of essential factors for the effectiveness and optimization of such a functional training. For example, it has become evident that, for an effective locomotor training of incomplete SCI subjects, the activation of load-and hip joint related afferent input is mandatory [12]. During the last years the application of advanced versions of robotic devices have been successfully established in functional training programmes. Such devices have the advantage that they allow for longer training times and can improve the training through virtual reality and feedback information technology [14].

The use of intrathecal baclofen therapy is well established in chronic spinal cord injured subjects. In a recent study, the incidence and management of tolerance in intrathecal baclofen therapy over a follow-up time of about 3 years is discussed [15]. On average the baclofen dose increases over the first 18 months and than stabilizes. Only about $20 \%$ of patients develop tolerance and about a third of patients require surgical revision of the pump system because of mechanical failures. Nevertheless, overall this therapy is suggested to be effective and safe, including over the long term.

\section{Regeneration-inducing therapies: promises and challenges}

For several years now, data from rodent studies indicate the feasibility of achieving partial repair of neuronal damage after a severe SCI. However, the success achieved in animal experiments often cannot be replicated in humans. In rodents, for example, the application of regeneration-facilitating olfactory ensheathing cells led to partial repair of spinal cord damage [16,17], while, in contrast, this approach (olfactory mucosal autografts) had no [18-20] or only little [21] effect on the neurological deficits in humans suffering severe SCI. During the last few years though, we have become increasingly aware of the problems associated with such translational studies [1,22]. There are several aspects of the complexity in this field which have to be considered.

First, the frequently used transection animal model for an SCI fits poorly with the human situation. In humans a contusional lesion with bleeding and edema usually extends over several segments of the spinal cord. Therefore, any functionally effective regeneration would certainly be harder to administer with benefit under these circumstances compared to the artificially induced experimental spinal tract transection.

Second, thoracic lesions are usually studied in animal models, whereas injury to the cervical cord (and second to the lumbar cord) is most common in humans [23]. However, in cervical and lumbar spinal cord contusions, the spinal damage concerns not only tract fibres, but also the motor neurones and their nerve roots over two or three segments 
which supply innervation to the arm and leg muscles, respectively. According to rodent experiments, up to $40 \%$ of the motor deficits might be attributed to the peripheral nervous system [24], and this would not be helped by an intervention directed at spinal tract regeneration.

Third, while acute animal models used for regenerationinducing therapies are well established, limited experience exists with subacute and chronic SCI. However, new interventional therapies are frequently applied to chronic spinal cord injured subjects in order to have a stable clinical condition [18, 20,21], and this typically occurs about a year after the initial trauma [23]. Studies with chronic, complete, SCI subjects during the last few years have provided evidence to suggest that the function of spinal neuronal circuits is impaired [25, 26]. However, a preservation of the function of spinal neuronal circuits below the level of lesion, is an essential prerequisite for the success of any kind of regeneration-inducing therapy [22]. Thus, even if regeneration of tract fibres is achieved, this may not necessarily be matched by a functional improvement. In addition, in chronic SCI, demyelination around the cavities [27] and scar formation [28] might prevent a significant amount of regeneration and so for most current studies, early intervention is recommended [22].

Fourth, most studies on regeneration-inducing interventions are applied in motor complete SCI subjects [18, 20, 21], because sensorimotor incomplete SCI subjects show a good spontaneous recovery of function [23, 29]. In a great number of these motor complete SCI subjects it has to be assumed that no more tissue bridges exist at the lesion site. However, for most of the presently available regeneration-inducing therapies (e.g., application of Nogo-antibodies) such tissue bridges are a prerequisite for successful tract fibre regeneration.

In any case, only a combination of regeneration-inducing therapies with functional training will allow for a better outcome of function, as it is only through this approach that appropriate connections with neuronal circuits can be established by the regenerating fibres.

In conclusion, although there has been much progress in translational medicine to repair the partially injured spinal cord, there still are challenges and open questions to be resolved before we can expect a successful application of regeneration-inducing therapies in SCI subjects. Nevertheless, by increasing our knowledge about the (dys)function of neuronal circuits in chronic SCI subjects, appropriate counter measures can hopefully be developed.

\section{References}

1. Dietz V (2008) Ready for human spinal cord repair? Brain 131(Pt.9):2240-2242
2. Wyndaele JJ (2009) Research related to spinal cord management is international. Spinal Cord 47(12):839-840

3. Debette $\mathrm{S}$ et al (2009) Long-term outcome of acute and subacute myelopathies. J Neurol 256(6):980-988

4. Leep Hunderfund AN, Wijdicks EF (2009) Intramedullary spinal cord hemorrhage (hematomyelia). Rev Neurol Dis 6(2): E54-E61

5. Petersen JA, Schubert M, Dietz V (2010) The occurrence of the Babinski sign in complete spinal cord injury. J Neurol 257(1): 38-43

6. Cheng MY et al (2009) Concomitant spinal cord and vertebral body infarction is highly associated with aortic pathology: a clinical and magnetic resonance imaging study. J Neurol 256(9): $1418-1426$

7. Ota K et al (2009) 18F-FDG PET successfully detects spinal cord sarcoidosis. J Neurol 256(11):1943-1946

8. Rath JJ, Ronday HK, Wirtz PW (2010) Acute transverse myelitis in psoriatic arthritis. J Neurol 257(3):457-458

9. Eger K et al (2010) Beevor's sign in facioscapulohumeral muscular dystrophy: an old sign with new implications. J Neurol 257(3):436-438

10. Taniguchi A et al (2010) Painful neck on rotation: diagnostic significance for crowned dens syndrome. J Neurol 257(1): 132-135

11. Matis GK, Birbilis TA (2009) Erythropoietin in spinal cord injury. Eur Spine J 18(3):314-323

12. Dietz V (2008) Body weight supported gait training: from laboratory to clinical setting. Brain Res Bull 76(5):459-463

13. Jakob W et al (2009) Difficulty of elderly SCI subjects to translate motor recovery- "body function"-into daily living activities. J Neurotrauma 26(11):2037-2044

14. Riener $\mathrm{R}$ et al (2010) Locomotor training in subjects with sensorimotor deficits: an overview of the robotic gait orthosis Lokomat. J Healthc Eng 1(2):197-216

15. Heetla HW et al (2009) The incidence and management of tolerance in intrathecal baclofen therapy. Spinal Cord 47(10): $751-756$

16. Barnett SC, Chang L (2004) Olfactory ensheathing cells and CNS repair: going solo or in need of a friend? Trends Neurosci 27(1):54-60

17. Ramon-Cueto A et al (2000) Functional recovery of paraplegic rats and motor axon regeneration in their spinal cords by olfactory ensheathing glia. Neuron 25(2):425-435

18. Chhabra HS et al (2009) Autologous olfactory mucosal transplant in chronic spinal cord injury: an Indian Pilot Study. Spinal Cord 47(12):887-895

19. Dobkin BH, Curt A, Guest J (2006) Cellular transplants in China: observational study from the largest human experiment in chronic spinal cord injury. Neurorehabil Neural Repair 20(1):5-13

20. Mackay-Sim A et al (2008) Autologous olfactory ensheathing cell transplantation in human paraplegia: a 3-year clinical trial. Brain 131(Pt 9):2376-2386

21. Lima $C$ et al (2010) Olfactory mucosal autografts and rehabilitation for chronic traumatic spinal cord injury. Neurorehabil Neural Repair 24(1):10-22

22. Dietz V, Curt A (2006) Neurological aspects of spinal-cord repair: promises and challenges. Lancet Neurol 5(8):688-694

23. Curt A et al (2008) Recovery from a spinal cord injury: significance of compensation, neural plasticity, and repair. J Neurotrauma 25(6):677-685

24. Collazos-Castro JE et al (2005) Motoneuron loss associated with chronic locomotion impairments after spinal cord contusion in the rat. J Neurotrauma 22(5):544-558

25. Dietz V et al (2009) Changes in spinal reflex and locomotor activity after a complete spinal cord injury: a common mechanism? Brain 132(Pt 8):2196-2205 
26. Dietz V, Muller R (2004) Degradation of neuronal function following a spinal cord injury: mechanisms and countermeasures. Brain 127(Pt 10):2221-2231

27. Guest JD, Hiester ED, Bunge RP (2005) Demyelination and Schwann cell responses adjacent to injury epicenter cavities following chronic human spinal cord injury. Exp Neurol 192(2):384-393

28. Klapka $\mathrm{N}$ et al (2005) Suppression of fibrous scarring in spinal cord injury of rat promotes long-distance regeneration of corticospinal tract axons, rescue of primary motoneurons in somatosensory cortex and significant functional recovery. Eur J Neurosci 22(12):3047-3058

29. Wirz $\mathrm{M}$ et al (2010) Outcome after incomplete spinal cord injury: central cord versus Brown-Sequard syndrome. Spinal Cord 48(5):407-414 